\title{
Comparative Histological and Immunohistochemical Study on The Effect of Curcumin and Atorvastatin in Induced Atherosclerosis in Aorta and Cardiac Muscle of Male Rabbits \\ Sheriff Aly Saleh ${ }^{1}$, Gamal Soliman Algharabawy ${ }^{1}$ and Mohamed Ghazy Attia Hablas ${ }^{2 *}$ \\ Department of Histology, Faculty of Medicine, Al-Azhar University, ${ }^{1}$ Cairo and ${ }^{2}$ Assiut, Egypt \\ *Correspondence author: Mohamed Ghazy Attia Hablas, Mobile: (+20) 01225958706, E-mail: drgazy78@ gmail.com
}

\begin{abstract}
Background: curcumin has many pharmacological effects including antioxidant, anti-inflammation, eliminating free radicals, anti-tumor, lipid regulation and anti-coagulation.

Objectives: to compare the histological effects of curcumin and atorvastatin in experimental induced atherosclerosis in the rabbit's aorta and heart.

Subjects and Methodology: thoracic aorta sections were prepared and stained by Hx\&E, orcein, Masson's trichrome and immuonohistochemical staning using CD34. Cardiac sections were prepared and stained by Hx\&E, Masson's trichrome stain and immuonohistochemical staning using troponin T. Morphometrical study included, the thickness of both tunica intima and media of thoracic aorta, area \% of the elastic fibers contents of both tunica intima and media in thorathic aorta, and the area \%of the immune reaction of CD34 in thoracic aorta .

Results: the atherosclerotic group showed a significant increase in the thickness of both tunicae intima\& media when compared with the control group. There was a significant decrease in elastic fibers content and significant increase in the mean area \% of the immune reaction of CD34 versus control. In atorvastatin treated group: there was a significant increase in comparison to control group, while there was a significant decrease in comparison to atherosclerotic group. There was significant decrease in elastic fibers content and significant increase in the mean area \% of the immune reaction of CD34 in comparison to control group. In curcumin treated group: the thickness of both tunica intima and media showed a significant increase in comparison to control group.
\end{abstract}

Conclusion: curcumin has potential role in treatment of atherosclerosis and myocardial infarction.

Keywords: Atherosclerosis, Atorvastatin, Curcumin, Aorta, Cardiac muscle.

\section{INTRODUCTION}

Atherosclerosis is potentially a life threatening disease develop characteristic atherosclerotic arterial plaque $^{(\mathbf{1})}$.

In atherosclerosis, fatty material accumulates in the intima with additional fibrosis ${ }^{(2,3)}$. This material is covered by dense fibrous tissue, which gives the plaque a white, pearly appearance ${ }^{(4)}$. These features result in the hardening of the arterial wall due to thickening and loss of elasticity. Hemorrhage, thrombosis, ulceration and calcification are advanced and late complicated lesions of atherosclerosis ${ }^{(5)}$.

Atorvastatin, is a member of the drug class known as statins, used for lowering blood cholesterol ${ }^{(5)}$. It also stabilizes plaque and prevents strokes through antiinflammatory and other mechanisms. Like all statins, atorvastatin works by inhibiting HMG-CoA reductase (3-hydroxy-3-methyl-glutaryl-CoA reductase). Curcumin is a polyphenolic compound and active constituent of the rhizomes of Curcuma longa plant from Zingiberaceae family ${ }^{(\mathbf{6})}$.

Curcumin is found to have many pharmacological effects including antioxidant, anti-inflammation, eliminating free radicals, anti-tumor, lipid regulation and anti-coagulation. Curcumin has extensive pharmacological anti-inflammatory, antioxidant, antimicrobial and cancer-preventive properties ${ }^{(7)}$.

It also has hypolipidemic and hypoglycemic properties. It is also reported that after application of curcumin in coronary heart disease, the lipid level is effectively controlled and the incidence of cardiovascular event is reduced. Controversial results have been observed in animal and human studies on lipid profile improvement by $\operatorname{curcumin}^{(8)}$.

Oxidative effect of curcumin results in preventive effect of curcumin on atherosclerotic development which can decrease lipid peroxidation and cholesterol levels of sera and tissues in mice and human stress and massive lipid deposition in the aorta ${ }^{(9)}$.

\section{MATERIALS AND METHODS}

\section{A- Materials:}

(1) Drugs:

1- Cholesterol powder $1 \%$ was purchased from Merck Company mixed with ordinary diet for a period of 8 weeks.

2- Atorvastatin trade name is Ator was purchased from EPICO pharmaceutical company in the form of tablets10mg.Each tablet was dissolved in $6 \mathrm{ml}$ saline. The rabbits of this group were given $5 \mathrm{mglkg}$ dissolved in $3 \mathrm{ml}$ saline solution orally by special oral syringe daily for 8 weeks.

3- Curcumin was purchased from spice dealer the recommended daily dose of curcumin is $100 \mathrm{mg} /$ $(\mathrm{kg} \cdot \mathrm{d})$ dissolved in $3 \mathrm{ml}$ saline solution daily for 8 weeks).

(II) The animal: The study was conducted at the animal house of Al-Azher University. The rabbit was the animal of choice because it is the most sensitive species for induction of atherosclerosis. New Zealand rabbit was the animal of choice in our study because its maximum life span is relatively short and well 
documented. The animal is easily caged and its diet could be easily controlled. Forty adult male rabbits were used throughout this study. The female rabbits were not used to avoid the effect of the cyclic physiologic hormonal changes. We used adult rabbits (6 week old rabbit) to avoid the sclerotic changes and other disorders and diseases which occur in old age. The average rabbit body weight used was about one kg.

Breeding: The animals were obtained from the Faculty of agriculture Al-A zher University according to the ethical guidelines for Care and Use of laboratory Animals. They were raised under hygienic condition in special housing boxes with food and water. They were fed on the standard rabbit food (standard rabbit pellets) which was free from cholesterol. The food was obtained from the Faculty of agriculture Al-Azher University.

The rabbits were divided into four groups each of ten animals each:

\section{Group I (Control group):}

This group included ten rabbits at the age of 6 weeks raised on the ordinary diet alone (without cholesterol or any antihypolipedemics) for the experimental period of 8 weeks.

\section{Group II (Atherosclerotic group):}

This group included ten rabbits at the age of 6 weeks raised on the ordinary diet mixed with $1 \%$ cholesterol powder for a period of 8 weeks ${ }^{(\mathbf{1 0})}$.

\section{Group III (atorvastatin treated atherosclerotic} group):

This group included ten rabbits at the age of 6 weeks raised on the ordinary diet mixed with $1 \%$ cholesterol powder and treated by atorvastatin $5 \mathrm{mg} \mathrm{kg}$ dissolved in saline solution daily at night for 8 weeks (11).

\section{Group IV (curcumin treated atherosclerotic group):}

This group included ten rabbits at the age of 6 weeks raised on the ordinary diet mixed with $1 \%$ cholesterol powder and treated by curcumin powder dissolved in saline solution daily for 8 weeks. The recommended daily dose of curcumin is $100 \mathrm{mg} /(\mathrm{kg} \cdot \mathrm{d})$ (12).

\section{B- Methods}

Rabbits representing the different groups were sacrificed under deep anesthesia. The chest wall was opened anteriorly and the two lungs were removed from their roots. The heart was carefully dissected and slinged from the apex and the thoracic aorta between the left subclavian artery and the diaphragm was carefully dissected. Both were freed from the overlying fat and perfused with saline to clean the champers of the heart and the luman of the aorta from the remaining blood. Then perfused with neutral formol for in situ fixation. The specimens, were rapidly removed and fixed in neutral buffered formol saline for preparation of paraffin sections ${ }^{(13)}$.

\section{Paraffin Sections:}

Specimens fixed in neutral buffered formal solution were left in the fixative for 48 hours, during that time several changes of the fixative were used every 12 hours. The specimens after being washed briefly in water were dehydrated in ascending grades of alcohol. Clearing was then carried out in benzene overnight. Specimens were then embedded in soft paraffin, then in hard paraffin in a plane perpendicular to the bottom of the block. Blocks containing pieces of the thoracic aorta and another blocks transverse section of the heart representing all experimental groups and control were prepared. The international rotatory microtome was used for cutting sections of 7 microns thickness ${ }^{(14)}$.

Sections of the control and experimental groups were mounted on slides and subjected to the following stains:

(1) Haematoxylin and eosin stain to study the histological structure of the wall of thoracic aorta and the myocardium ${ }^{(\mathbf{1 5})}$.

(2) Modified Taenzer-Unna Orcein method to study the elastic fibers present in the aorta ${ }^{(16)}$.

(3) Masson trichrome technique, to study the collagen fibers in ghe heart muscle and smooth muscles in the aorta ${ }^{(17)}$

(4) Immunohistochemical Technique: -CD34 \&Troponin $\mathrm{T}^{(\mathbf{1 8})}$.

\section{Haematoxylin \& Eosin ${ }^{(14)}$ Reagents}

(1) Harris Haematoxylin Solution: Haematoxylin(1.0gm.), Absolute alcohol(10 ml.), Distilled water $(190 \mathrm{ml}$.), Aluminium potassium sulphate (20 gm.), Mercuric oxide (0.5 gm.), Glacial acetic acid $(0.8 \mathrm{ml}$.)

(2) Eosin Solution: Eosin (standard stain, water soluble)(1.0 gm.),Distilled water (100 ml.), Eosin was dissolved in water.

Results: Nuclei (Blue), Cytoplasm According to the reaction of the cytoplasm (pink or blue)

Orcein

Reagents: Orcein (1.0 gm); 80\% Ethyl alcohol (100 $\mathrm{ml}$.) Concentrated hydrochloric acid $(1.0 \mathrm{ml}$.)

Results: Elastic fibers (brown) ${ }^{(23)}$.

\section{Masson Trichrome}

Reagents: Ponceau-acid Fuchsin solution, 1\% aqueous phosphomolybdic acid, $2 \%$ light green in $2 \%$ acetic acid diluted 12: 10 with distilled water prior to use.

Acid Fuchsin Solution: Acid fuchsin (1.0 gm.), Distilled water (100 ml.), Trichloracetic acid (3.0 gm.).

- Ponceau Solution: Ponceau de Xylidine (1.0 gm.), Distilled water (100 ml.), Acetic acid (1.0 ml.)

Results:

-Collagen fibers (Green), Smooth muscles (Red), Nuclei (Blue) ${ }^{(19)}$. 
Immunohistochemical method

\section{Immunohistochemical staining for CD34 and troponin $\mathbf{T}$ \\ Primary antibody}

1- CD34 (Endothelial cell marker) Ab-1 (Clone QBnd/10)

It is a mouse Monoclonal Antibody (Lab Vision Corporation laboratories, CA 94539, USA, catalogue number MS-363-R7). It was supplied as $(7.0 \mathrm{ml})$ of antibody $(200 \mathrm{ug} / \mathrm{ml})$ prediluted in $0.05 \mathrm{~mol} / \mathrm{L}$ Tris$\mathrm{HCL}, \mathrm{Ph} 7.6$ containing stabilizing protein and $0.015 \mathrm{~mol} / \mathrm{L}$ sodium azide. It was stored at $2-8 \mathrm{C}$. No special pretreatment is required for immunehistochemical staining of formalin-fixed tissues.

2- Troponin T (Cardiac muscle marker) Ab-1, Clone: 13-11:

It is a mouse monoclonal Antibody (Lab Vision Corporation laboratories, USA, catalogue number MS295-P0). It was supplied as $(7.0 \mathrm{ml})$ of antibody (200ug/ml) purified from the ascites fluid by protein $\mathrm{G}$ chromatography, prepared in $10 \mathrm{~m} \mathrm{M} \mathrm{PBS,} \mathrm{Ph} \mathrm{7.4,} \mathrm{with}$ $0.09 \%$ sodium azid. It was stored at 2-8 C. Staining of formalin-fixed tissue required boiling tissue sections in $10 \mathrm{mM}$ citrate buffer, Ph6.0, for 10-20 minutes followed by cooling at room temperature for 20 minutes.

\section{Detection system for antibodies:}

Histostatin SP kit (LAB-SA system, zymed laboratories Inc, San Francico, CA 94080, USA, catalogue number 95-9643). It is a broad spectrum detection system that reacts with mouse, rabbit, guinea pig and rat primary antibody. This Histostatin SP kit includes serum blocking solution, a biotinylated secondary antibody, a horse radish peroxidase streptavidin and a substrate chromogen mixture to demonstrate antigen in cells and tissues.

\section{Reagent supplied in this kit water :}

- Reagent $1 \mathrm{~A}$, one dropper bottle $(15 \mathrm{ml})$ of ready -to use serum blocking solution, $10 \%$ non- immune serum (goat).

- Reagent $1 \mathrm{~B}$, one dropper bottle $(15 \mathrm{ml})$ of ready -to use serum biotinylated secondary antibody.

- Reagent 2, one dropper bottle (15 ml) of ready -to - use serum streptavidin-peroxidase conjugate.

- Reagent 3A, one dropper bottle $(3 \mathrm{ml})$ of concentrated substrate buffer.

- Reagent 3B, one dropper bottle $(3 \mathrm{ml})$ of concentrated chromogen solution DAB (diaminobenzedine).

- Reagent $3 \mathrm{C}$, one dropper bottle $(3 \mathrm{ml})$ of $6 \%$ hydrogen peroxide.

- Reagent 4 , one dropper bottle $(15 \mathrm{ml})$ of ready -to use- hematoxylin.

- Reagent 5, one dropper bottle $(15 \mathrm{ml})$ of ready -to use histomuont.
Data obtained was statistically analyzed:

Data were tabulated and statistically analyzed to evaluate the difference between the groups under study as regards the various parameters. Together, correlations were tried in between the essential studied parameters. The statistical analysis included; the arithmetic mean, standard deviation, analysis of variance (ANOVA). ANOVA was performed to test for the significance of difference between means of all studied groups using one-way ANOVA test \&post-Hoc Multiple Comparisons test. The probability $(\mathrm{P})$ value obtained from statistical tables was directly supplied by the computer using statistical package for social sciences (SPSS) software version 16. Results were considered statistically significant when $\mathrm{p}$ was $<0.05$.

\section{RESULTS}

Evaluation of the changes in the aorta and heart of control and experimental groups were done through examination of sections by light microscope and image analyzer. The following changes were found and recorded.

\section{Histological result of aortic sections:}

1-Hematoxylin and Eosin stained aortic sections:

\section{A. Control group:}

Histological examination of thoracic aorta sections in the control group showed intact tunica intima with thin wavy corrugated endothelium, thin sub endothelial layer and apparent wavy corrugated internal elastic fibers. Smooth muscle and elastic fibers make up the tunica media. Underneath is the tunica adventitia made of loose connective tissue (Figs.1\&2).

\section{B. Atherosclerotic group:}

In the atherosclerotic group the tunica intima showed absent corrugation with apparent bulge formation. There were adhesions of RBCs on the surface of the intima. The intima showed vacuolated cells in the sub endothelial layer probably occupied by fatty material (foam cell). Unlike the control, the subintima was wide. There was apparent increase in the thickness of both tunicae intima \& media when compared with the control group (Figs. 3\&4).

\section{Atorvastatin treated group:}

In Atorvastatin treated group the tunica intima was intact with thin wavy corrugated endothelium while some areas possessed absence of such corrugation .Thin sub endothelial layer and apparent wavy corrugated internal elastic lamina. Smooth muscle and elastic fibers make up the tunica media. Underneath is the tunica adventitia made of loose connective tissue (Figs. 5\&6).

\section{Curcumin treated group:}

In curcumin treated group there was intact tunica intima with thin wavy corrugated endothelium, thin sub endothelial layer and apparent wavy corrugated internal elastic lamina. Smooth muscle and elastic 
fibers make up the tunica media. Underneath is the tunica adventitia made of loose connective tissue (Figs.7\&8).

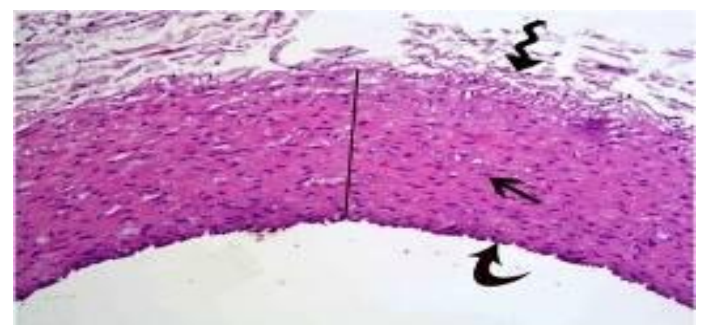

Fig. (1): A photomicrograph in a section of a thoracic aorta in the control group showing tunica intima (curved arrow) faces the lumen. Smooth muscle and elastic fibers make up the tunica media (arrow). Underneath is the tunica adventitia made of loose connective tissue (spiral arrow). Note the thickness of both tunicae intima \& media (straight line). (Haematoxylin\& Eosin x200)

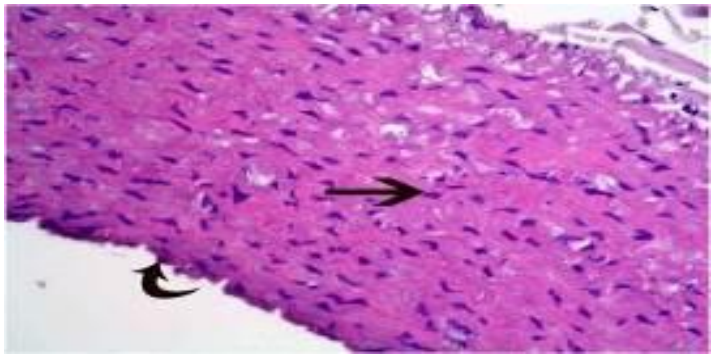

Fig. (2): A photomicrograph in a section of a thoracic aorta in the control group showing tunica intima(curved arrow) with thin wavy corrugated endothelium and thin sub endothelial layer. Smooth muscle and elastic fibers make up the tunica media (arrow).

(Haematoxylin\& Eosin $x 400$ )

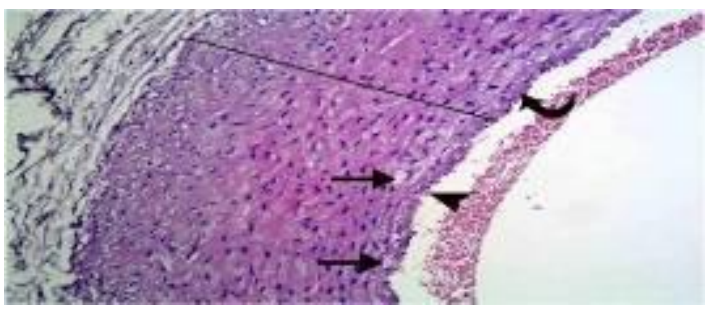

Fig. (3): A photomicrograph in a section of a thoracic aorta in the atherosclerotic group showing tunica intima with no corrugation and apparent bulge formation (curved arrow) as well as adhesions of RBCs on the surface of the intima (arrow head). Note the presence of vacuolated cells in the sub endothelial layer(foam cell) (arrows). There is apparent increase in the thickness of both tunicae intima \& media (straight line). (Haematoxylin\& Eosin x200)

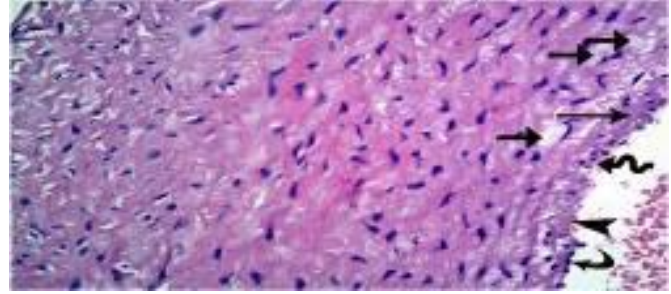

Fig.(4): A photomicrograph in a section of a thoracic aorta in the atherosclerotic group showing tunica intima absent corrugation (curved arrow) .Adhesions of RBCs on the surface of the intima (arrow head).Note the presence of many foam cells in sub endothelial layer (arrows).There is apparent increase in the thickness of the sub endothelial layer (thin arrow).Also there are many mononuclear cell infiltration in the tunica intima(spiral arrow).

\section{(Haematoxylin\& Eosin x400)}

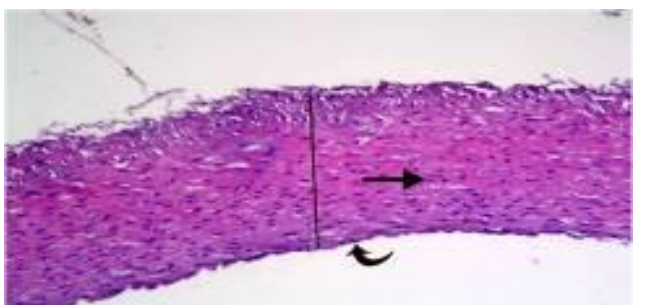

Fig. (5): A photomicrograph in a section of a thoracic aorta in the Atorvastatin treated group showing some areas of tunica intima possessing lost corrugation (curved arrow). Smooth muscle and elastic fibers make up the tunica media (arrow). Note the thickness of both tunicae intima \& media (straight line). (Haematoxylin\& Eosin x200)

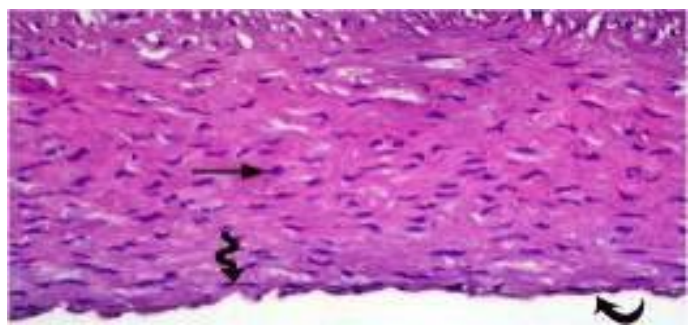

Fig. (6): A photomicrograph in a section of a thoracic aorta in the atorvastatin treated group showing tunica intima with lost corrugation (curved arrow) other areas appeared wavy (spiral arrow). Smooth muscle and elastic fibers make up the tunica media (arrow). (Haematoxylin\&Eosinx400)

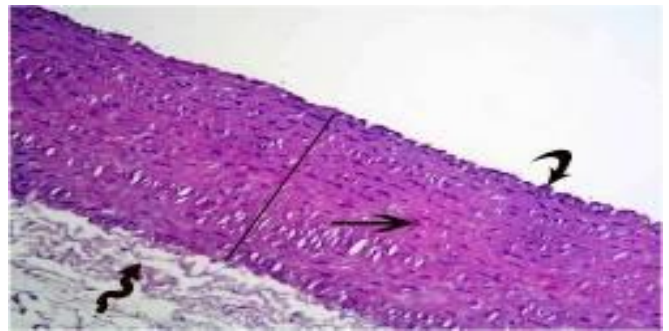

Fig. (7): A photomicrograph in a section of a thoracic aorta in the curcumin treated group showing intact tunica intima(curved arrow) faces the lumen. 
Smooth muscle and elastic fibers make up the tunica media (arrow). Underneath is the tunica adventitia formed of loose connective tissue (spiral arrow) . Note the thickness of both tunicae intima \&media (straight line).

(Haematoxylin\& Eosin x200)

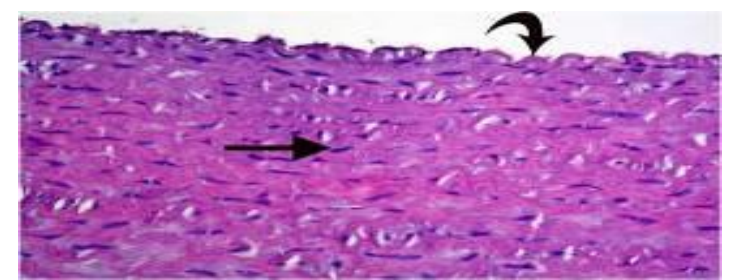

Fig. (8): A photomicrograph in a section of a thoracic aorta in the curcumin treated group showing intact tunica intima(curved arrow) with thin wavy corrugated endothelium and thin sub endothelial layer. Smooth muscle and elastic fibers make up the tunica media (arrow). (Haematoxylin\& Eosin $\mathbf{4 0 0 )}$

\section{2- Orcein stained aortic sections}

\section{A. Control group:}

Histological examination of (orcein) stained sections of thoracic aorta of the control group showed prominent wavy internal elastic lamina. Tunica media appears rich in elastic fibers. These fibers are parallel in distribution (Fig. 9).

\section{B. Atherosclerotic group:}

Atherosclerotic group showed apparently interrupted internal elastic lamina. Tunica media possessed areas with complete lose of elastic fibers. Other areas showed irregularly condensed elastic fibers towards the tunica adventitia (Fig.10).

\section{Atorvastatin treated group:}

Atorvastatin treated group showed prominent intact internal elastic lamina. Tunica media appeared rich in parallel elastic fibers. Small areas showed lost elastic fibers and prominent external elastic lamina (Fig.11).

\section{Curcumin trated group:}

Curcumin treated group showed prominent intact internal elastic lamina. Tunica media appeared rich in parallel elastic fibers. Small areas showed lost elastic fibers and prominent external elastic lamina (Fig.12).

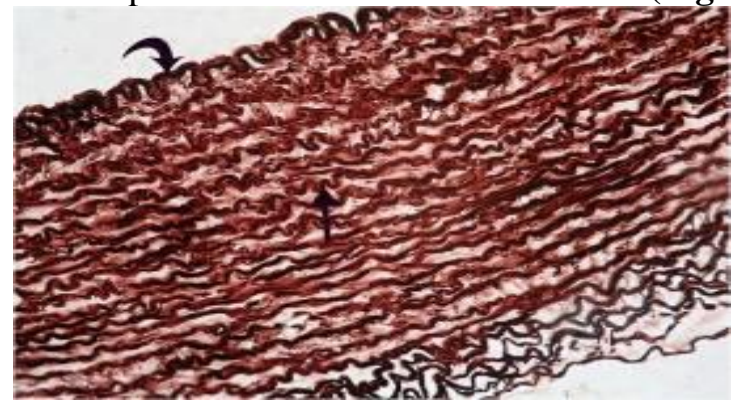

Fig. (1): A photomicrograph in a section of a thoracic aorta in the control group showing prominent dark brown wavy internal elastic lamina (curved arrow) .Tunica media appears rich in elastic fibers. These fibers are parallel in distribution (arrow)

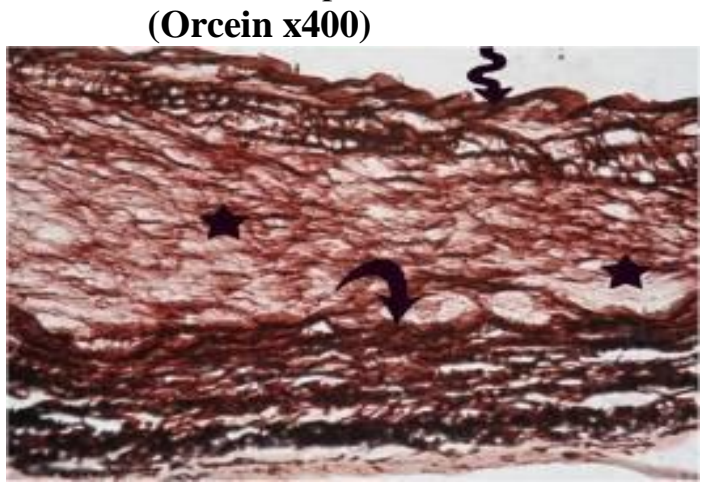

Fig.(10): A photomicrograph in a section of a thoracic aorta in the atherosclerotic group showing apparently interrupted internal elastic lamina (spiral arrow) .Tunica media possesses areas with complete lose of elastic fibers(stars). Other areas show irregularly condensed elastic fibers to words the tunica adventitia (curved arrow).

(Orcein $x 400)$

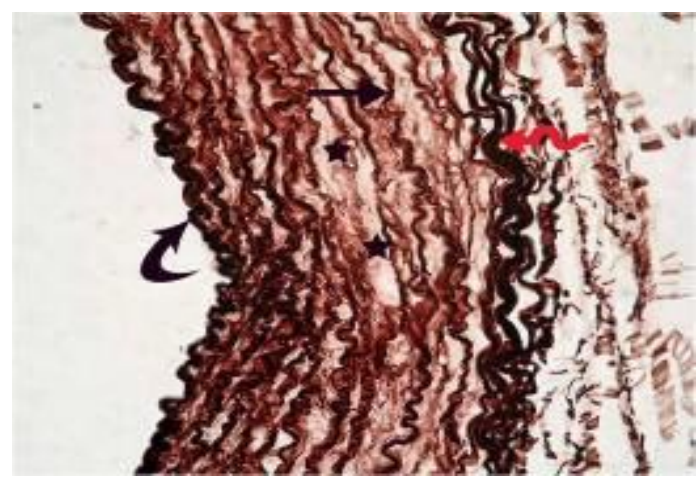

Fig. (2): A photomicrograph in a section of a thoracic aorta in the atorvastatin treated group showing prominent intact internal elastic lamina (curved arrow). Tunica media appears rich in parallel elastic fibers (arrow). Small areas showed lost elastic fibers (stars). Note the presence of prominent external elastic lamina (red spiral arrow). (Orcein x400)

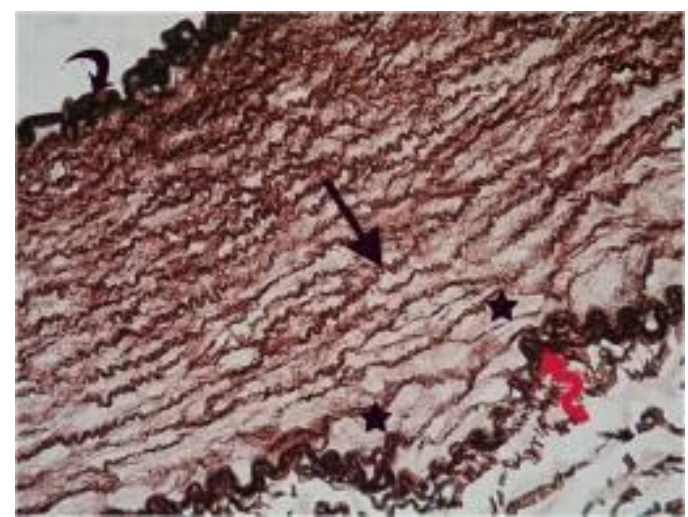

Fig. (12): A photomicrograph in a section of a thoracic aorta in curcumin treated group showing prominent intact internal elastic lamina (curved arrow). Tunica media appears rich in parallel elastic fibers (arrow). Small areas showed lost elastic fibers 
(stars).Note the presence of prominent external elastic lamina (red spiral arrow).

(Orcein x400)

\section{3-Masson's trichrome stained aortic sections:}

\section{A. Control group}

Masson's trichrome stained sections of a thoracic aorta showed wavy elastic fibers and smooth muscle cells distributed in between (Fig.13).

\section{B. Atherosclerotic group}

Atherosclerotic group showed smooth muscle fibers in the tunica intima most probably migrated from the tunica media. There was apparent decrease in amount of smooth muscles in the tunica media and disorganized elastic fibers (Fig.14).

\section{Atorvastatin treated group}

Atorvastatin treated group showed some smooth muscle fibers in the tunica intima. The tunica media possessed wavy elastic fibers and smooth muscle fibers distributed in between (Fig.15).

\section{Curcumin treated group}

Curcumin treated group showed wavy elastic fibers and smooth muscle cells distributed in between (Fig.16).

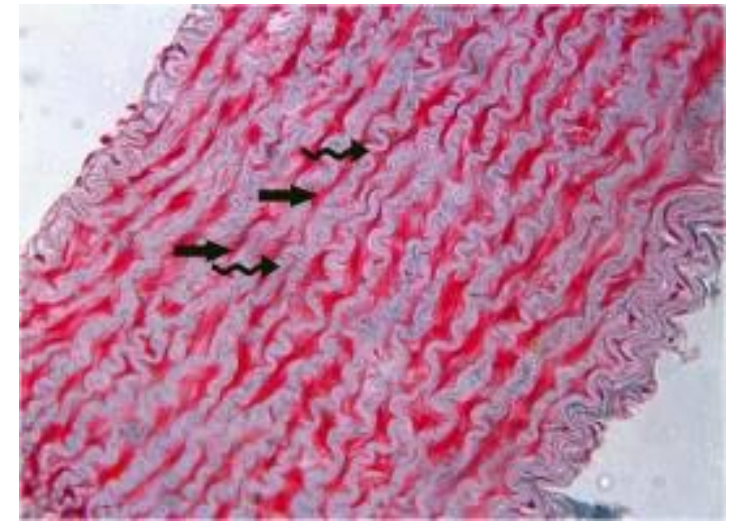

Fig. (3): A photomicrograph in a section of a thoracic aorta in Control group showing wavy elastic fibers (spiral arrows) and smooth muscle cells distributed in between (arrows). (Masson's trichrome $x$ 400)

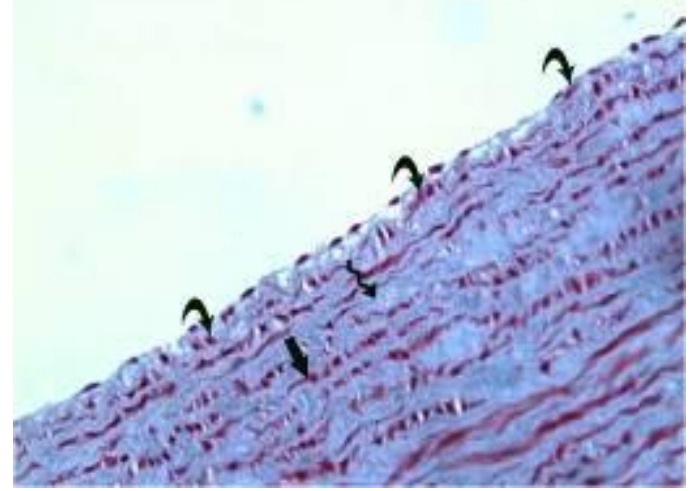

Fig.(4):A photomicrograph in a section of a thoracic aorta in Atherosclerotic group showing smooth muscle fibers in the tunica intima (curved arrows ),apparent decrease in amount of smooth muscles in the tunica media (arrow) and disorganized elastic fibers (spiral arrow).

(Masson's trichrome $x$ 400)

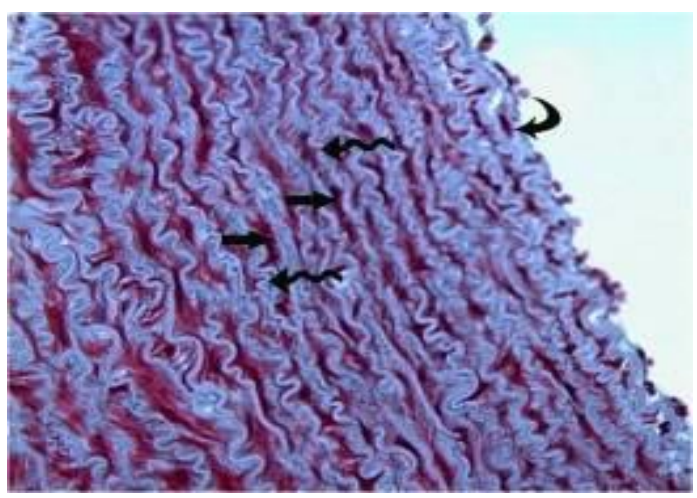

Fig. (15): A photomicrograph in a section of a thoracic aorta in atorvastatin treated group showing some smooth muscle in the tunica intima (curved arrow).Note the presence elastic fibers (spiral arrows)in between smooth muscle in the tunica media (arrows).

(Masson's trichrome $x$ 400)

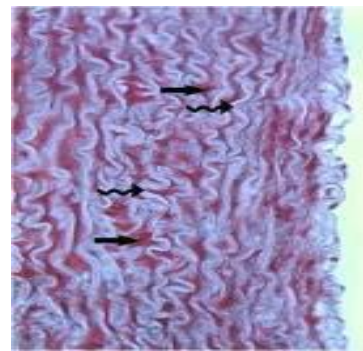

Fig. (16): A photomicrograph in a section of a thoracic aorta in curcumin treated group showing wavy elastic fibers (spiral arrows) and smooth muscle cells distributed in between (arrows). (Masson's trichrome $x$ 400)

\section{4- CD34 immunohistocemical stained aortic} sections:

\section{A. Control group}

This group recorded few positive brownish cytoplasmic reaction confined to endothelial cells (Fig.17).

\section{B. Atherosclerotic group}

This group showed many strong positive CD34 immuonostaining confined to cytoplasm endothelial cells was observed (Fig.18).

\section{Atorvastatin treated group}

This group showed many strong positive CD34 immuonostained endothelial cells (Fig.19).

\section{Curcumin treated group}

Few moderate CD34 immunoreaction was detected in cytoplasm of endothelial cells (Fig.20). 


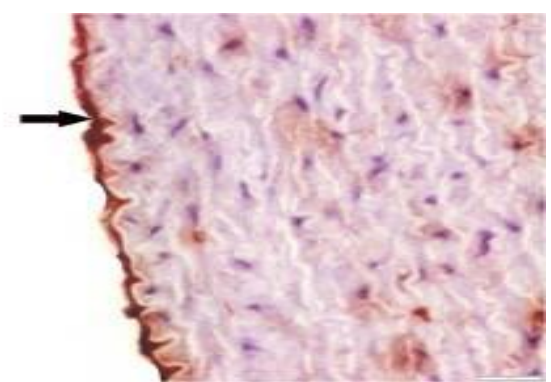

Fig. (17): A photomicrograph in a section of a thoracic aorta in control group showing few positive CD34 immuonostaning confined to the cytoplasm of endothlial cells(arrow). (Anti CD34 immuonostaning $x$ 400)

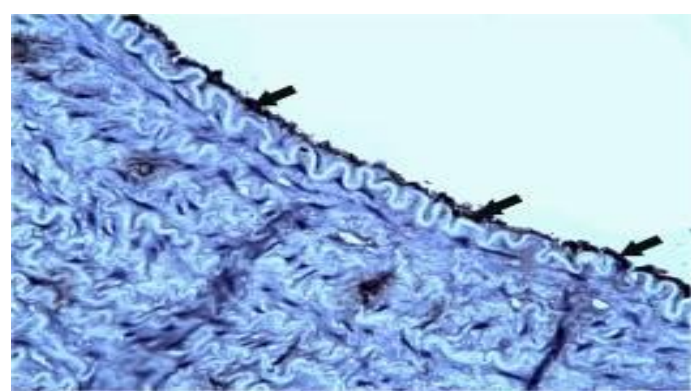

Fig. (18): A photomicrograph in a section of a thoracic aorta in atheroscleroticl group showing many strong positive CD34 immuonostaning confined to cytoplasm of endothlial cells (arrows). (Anti CD34 immuono staning $x$ 400)

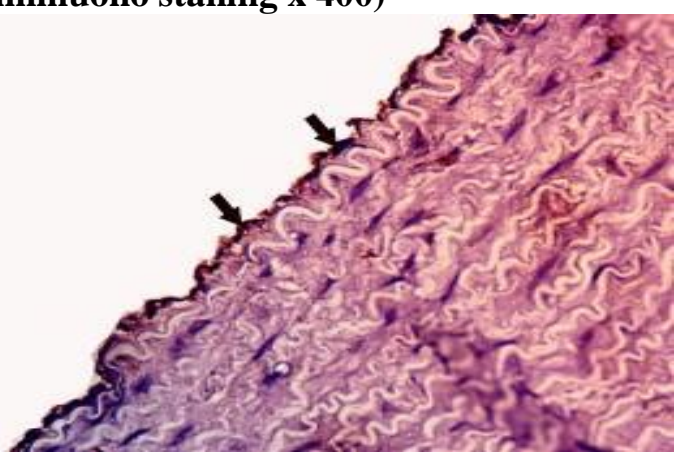

Fig. (19): A photomicrograph in a section of a thoracic aorta in Atorvastatin treated group showing strong positive CD34 immuonostaning confined to cytoplasm endothlial cells(arrows). (Anti CD34 immuono staning $x$ 400)

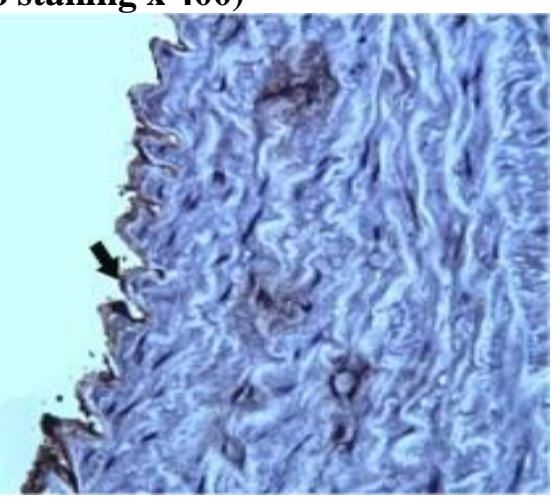

Fig. (20): A photomicrograph in a section of a thoracic aorta in curcumin treated group showing few moderate positive CD34 immuonostaning confined to cytoplasm endothlial cells(arrows). (Anti CD34 immuono staning $x$ 400)

\section{Morphometric results of aorta}

1- Mean thickness of both tunica intima and media of thoracic aorta:

In Hematoxylin and Eosin stained aortic sections, the mean thickness of both tunica intima and media of group 1 (control group) was $208+6.69$.There was an increase in the thickness in atherosclerotic group , atorvastatin treated group and in curcumin treated group to be $294.1+4.39,273+9.15$ and $248+6$ respectively (table 1).

There was a significant increase in the thickness of both tunica intima and media in all studied groups in comparison to control group. Mean while, there are significant decrease in the thickness both tunica intima and media in atorvastatin and curcumin treated groups in comparison to atherosclerotic group (Fig 21).

Table (1): The Mean thickness of tunica intima\& media in all studied groups

\begin{tabular}{|c|c|c|c|c|}
\hline & Group I & Group II & Group III & Group IV \\
\hline $\begin{array}{c}\text { Mean } \\
\text { thickness } \\
\text { of tunica }\end{array}$ & 208 & $294^{*}$ & $273^{* \#}$ & $248^{* \#}$ \\
$\begin{array}{c}\text { intima\& } \\
\text { media }\end{array}$ & 6.6 & 4.39 & 9.15 & 6 \\
\hline
\end{tabular}

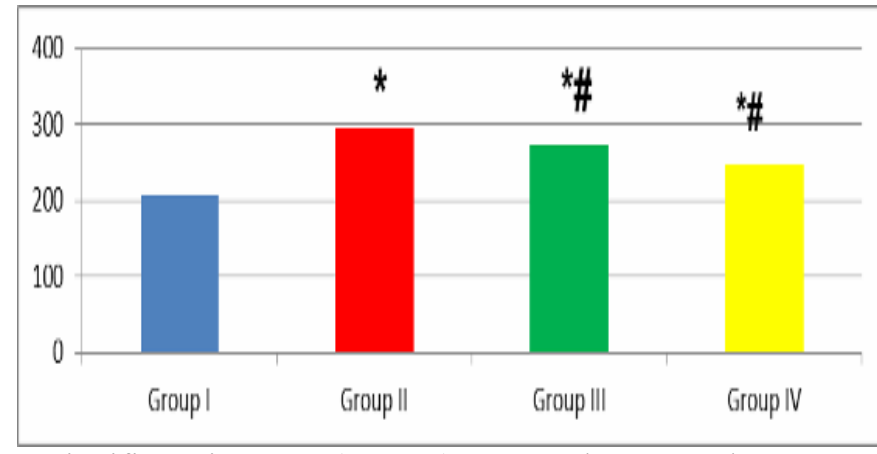

* Significant increase $(\mathrm{P}<0.05)$ compared to control *\# Significant decrease $(\mathrm{P}<0.05)$ compared to group II

Fig. (5): Mean thickness of tunica intima \&tunica media in all studied groups

\section{2- Mean area \%of the elastic fibers contents in thorathic aorta}

Mean area $\%$ of the elastic fibers in thorathic aorta stained by orcein of group 1( control group ) was $34.65+8.85$, while there was decrease in the mean area\% of elastic fibers in all other groups as follows $12.09+3.35,22.37+6$ and $29.31+6.28$ respectively in atherosclerotic, atorvastatin and curcumin treated groups (Table 2).

There is significant decrease in elastic fibers content in atherosclerotic and atorvastatin treated group in comparison to control group while there was 
no significant difference between curcumin treated group and control group (Fig.22).

Table (2): The mean area \%of elastic fibers in all studied groups

\begin{tabular}{|c|c|c|c|c|}
\hline & Group I & Group II & Group III & Group IV \\
\hline $\begin{array}{c}\text { Mean area \% } \\
\text { of elastic } \\
\text { fibers }\end{array}$ & 34.65 & $12.09^{*}$ & $22.37 *$ & 29.31 \\
\hline
\end{tabular}

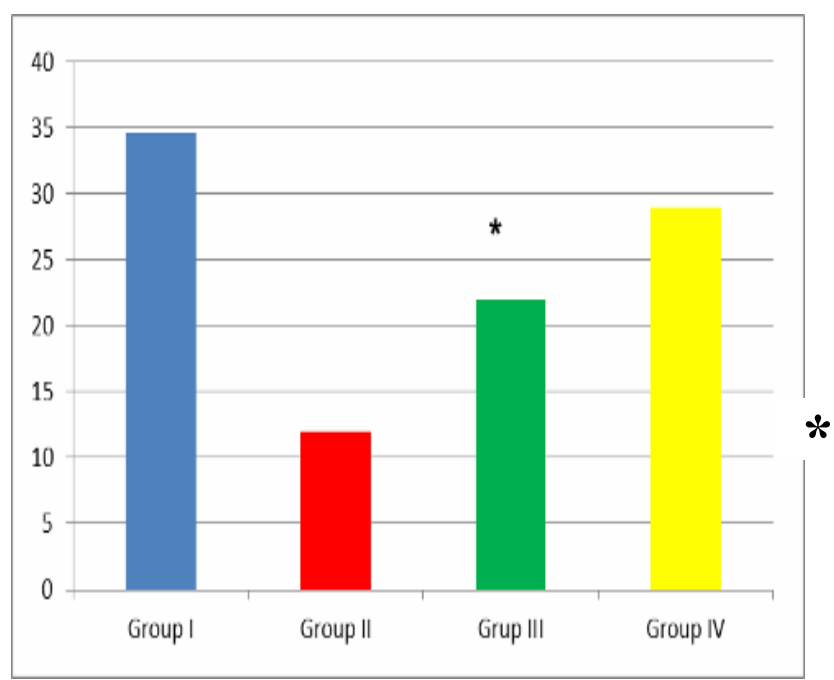

* Significant decrease $(\mathrm{P}<0.05)$ compared to control

Fig.( 6): Mean area\% of elastic fibers in the aorta of all studied groups stained by orcien.

\section{3- Mean area \%of the immune reaction of CD34}

The mean area $\%$ of immunoreactive endothelial cell of thoracic aorta stained by CD34 immuonohistochemical stain of control group was $1.8+$ 1.1. There was an increase in the mean area\% of immunopostive endothelial cells in atherosclerotic group, atorvastatin treated group and in curcumin treated group to be $10.66+2.4,5.27+1.7$ and $2.6+0.8$ respectively (Table 3).

There was a significant increase in the mean area \% of the immune reaction of CD34 in group II and group III in comparison to control group and was no significant difference between curcumin treated group and control group (Fig.23).

Table (3): The mean area \% immune reaction of CD34 in all studied groups

\begin{tabular}{|c|c|c|c|c|}
\hline & $\begin{array}{c}\text { Group } \\
\text { I }\end{array}$ & $\begin{array}{c}\text { Group } \\
\text { II }\end{array}$ & Group III & Group IV \\
\hline $\begin{array}{c}\text { Mean area\% } \\
\text { immune } \\
\text { reaction of } \\
\text { CD34 }\end{array}$ & 1.8 & $10.66^{*}$ & $5.27 *$ & 2.6 \\
\hline
\end{tabular}

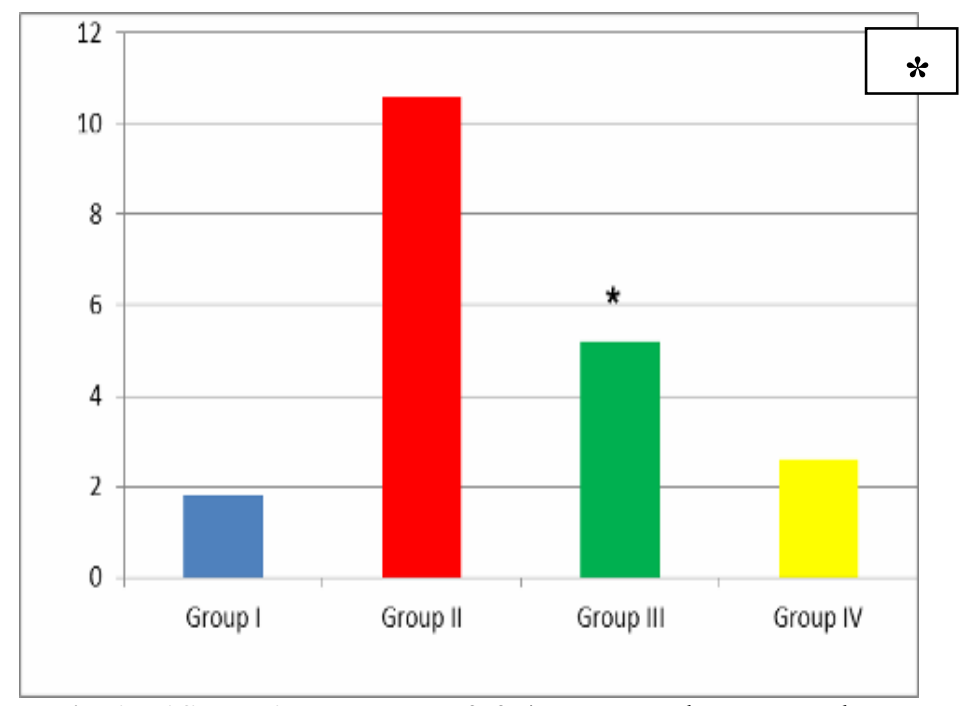

* Significant increase $(\mathrm{P}<0.05)$ compared to control

Fig. (7):Mean area\% immune reaction of CD34 in the aorta of all studied groups

\section{Histological result of cardiac muscle sections: 1-Hematoxylin and Eosin stained sections: \\ A. Control group:}

Histological examination of cardiac muscle sections in the control group showed branching cardiac muscle fibers with centrally located nuclei. Each muscle fiber is surrounded with endomysium containing blood vessels and cells with flat nuclei most probably fibrocyte (Figs.24\&25).

\section{B. Atherosclerotic group:}

Atherosclerotic group showed many muscle fibers with pale cytoplasm and darkly stained peripheral nuclei. Many cardiac muscle fibers appeared darkly acidophilic with darkly stained nucleus, while others possessed apoptotic change in the form of vacuolated cytoplasm with small dark (pyknotic nuclei). There were mononuclear infiltration and thickened media of a blood vessel with formation of bulge towords the lumen to which RBCs were stuck. There was wide endomysium between cardiac muscle fibers (Figs.26\&27).

\section{Atorvastatin treated group:}

Atorvastatin treated group showed most of cardiac muscle fibers with vacuolated and small dark (pyknotic) nuclei .There was wide endomysium space between cardiac muscle fibers. RBCs were stuck on the lumen of blood vessel (Fig.28).

\section{Curcumin trated group:}

Curcumin treated group showed many healthy branching cardiac muscle fibers with pale central nucleus. Many cardiac muscle fibers possessed pale cytoplasm with absent nuclei, while others showed vacuolated cytoplasm with pycnotic nuclei. RBCs were stuck on the lumen of blood vessel (Figs.29\&30). 


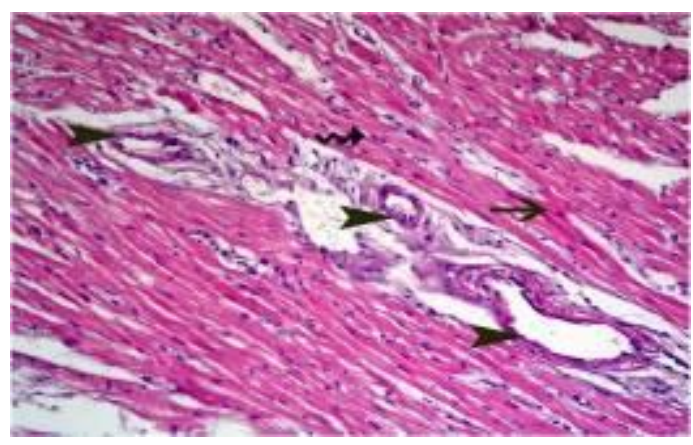

Fig. (24): A photomicrograph of a section of heart from group I (control group) showing branching cardiac muscle fibers with centrally located nuclei (arrow).Each muscle fiber is surrounded with endomysium containing cells with flat nuclei (spiral arrow). Note the presence of blood vessels (arrow heads) (H\&Ex 200)

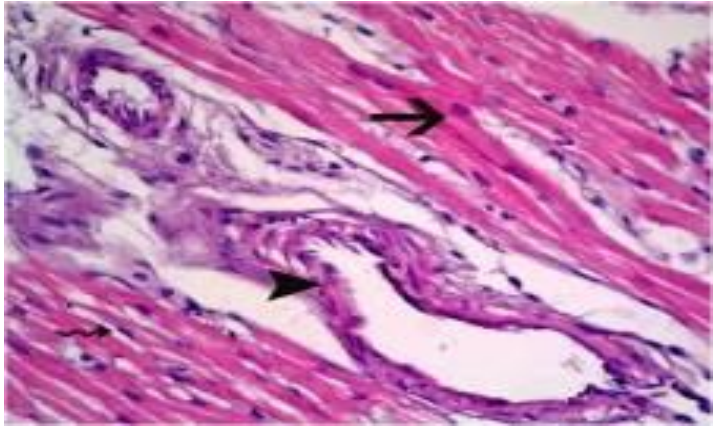

Fig.(25 ): A photomicrograph of a section of heart from group I (control group) showing branching cardiac cell with centrally located nuclei (arrow).Each muscle fiber is surrounded with endomysium containing cells with flat dark nuclei (spiral arrow). Note the presence of blood vessels (arrow head) with patent lumen.

\section{(H\&E x400)}

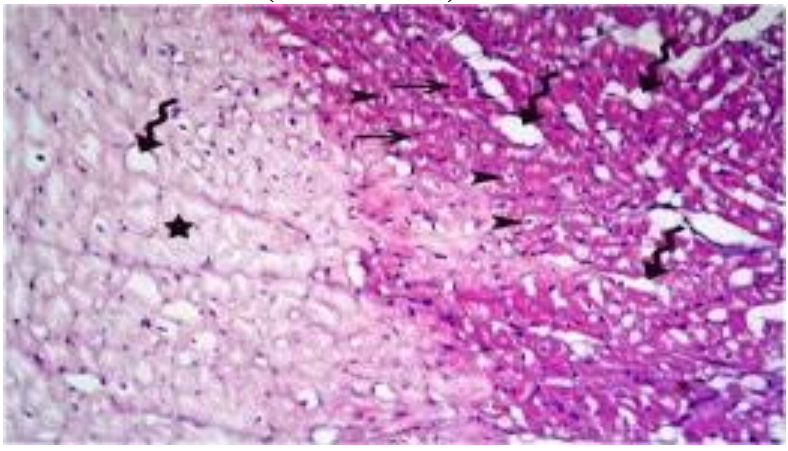

Fig.(26): A photomicrograph of a section of heart from group II (atherosclerosis group) showing many muscle fibers with pale cytoplasm and darkly stained peripheral nuclei(star).Many cardiac muscle fibers appear darkly acidophilic with darkly stained nucleus (arrows), while others possess apoptotic change in the form of vacuolated cytoplasm with small dark (pyknotic nuclei) (arrow heads). Note the presence of wide space between cardiac muscle fibers (spiral arrows).

(H\&EX200)

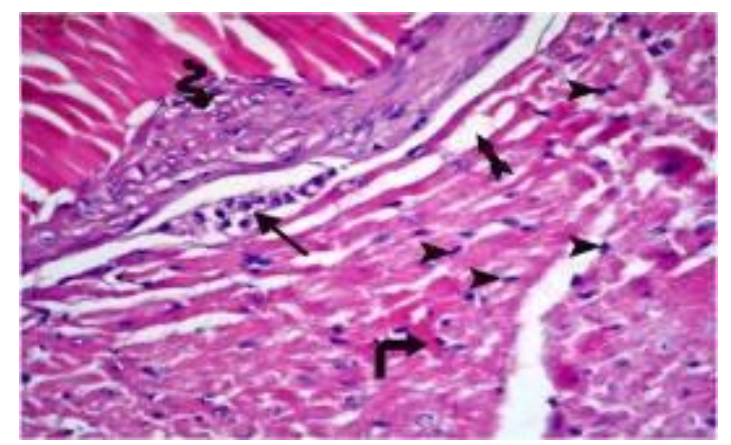

Fig.(8): A photomicrograph of a section of heart from group II (atherosclerosis group) many cardiac muscle fibers appear darkly acidophilic with darkly peripheral nucleus (curved arrow), while others possess dark (pyknotic nuclei) (arrow heads) . There are mononuclear infiltrations (arrow). Note the thickened media of a blood vessel with formation of bulge towards the lumen to which RBCs stick (spiral arrow). Note the presence of wide space between cardiac muscle fibers (arrows with bifid tail).

(H\&EX400)

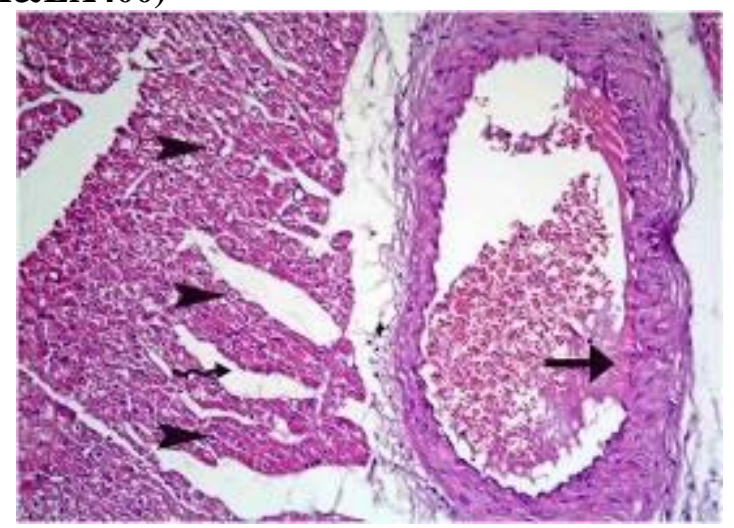

Fig.(28): A photomicrograph of a section of heart from group III (Atorvastatin treated group) showing most of the cytoplasm of cardiac muscle fibers are vacuolated with small dark (arrow heads). Note the presence of wide endomethyium (spiral arrow). Note the stickiness of RBCS on the lumen of blood vessel (arrow). (H\&Ex200)

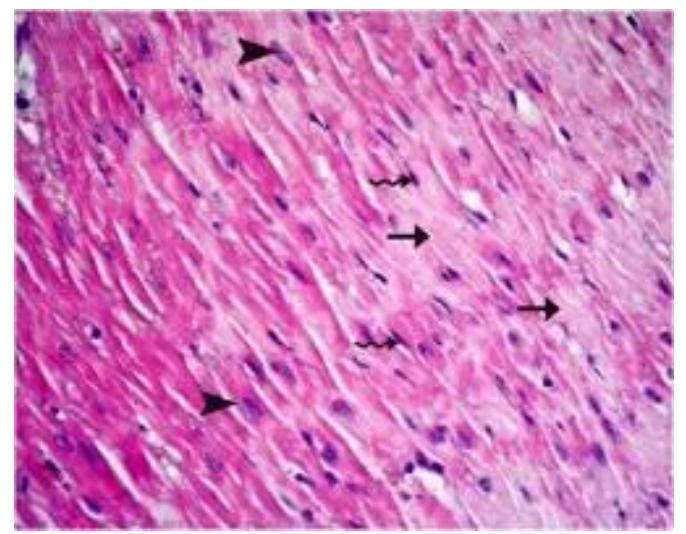

Fig.(29): A photomicrograph of a section of heart from group IV (curcumin treated group) show many healthy branching cardiac muscle fibers with pale central nucleus (arrow heads). Many cardiac muscle fibers possess pale cytoplasm with absent nuclei 
(arrows), while others show dark peripheral nuclei (spiral arrows).

\section{(H\&EX200)}

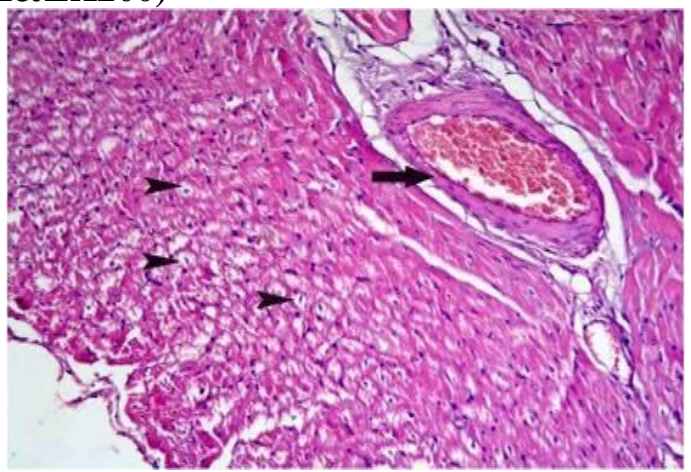

Fig.(30): A photomicrograph of a section of heart from group IV (curcumin treated group) showing many muscle fibers with vacuolated cytoplasm with small dark (arrow heads).Note the stickiness of RBCS on the lumen of blood vessel. (H\&EX400)

2- Masson's trichrome stained cardiac muscle sections:

\section{A. Control group}

Sections of cardiac muscle stained by Masson's trichrome showed minimal collagen fibers deposition around the blood vessels (Fig.31).

\section{B. Atherosclerotic group}

Examination of sections from heart from group II (atherosclotic group) showed dense collagen fibers deposition around blood vessels (Fig.32) and in between interrupted cardiac muscle fiber (Fig.33).

\section{Atorvastatin treated group}

Atorvastatin treated group showed dense collagen fibers deposition around blood vessels (Fig.34).

\section{Curcumin treated group}

Curcumin treated group showed minimal collagen fibers deposition in between cardiac muscle fibers (Fig.35).

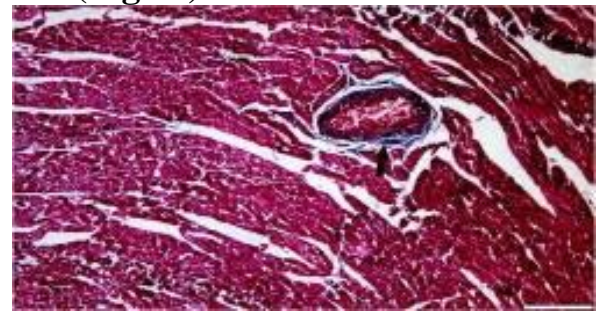

Fig.(31): A photomicrograph of a section of heart from group I (control group) showing minimal collagen fibers around blood vessels(arrow). (Masson's

\section{trichrome $x$ 400)}

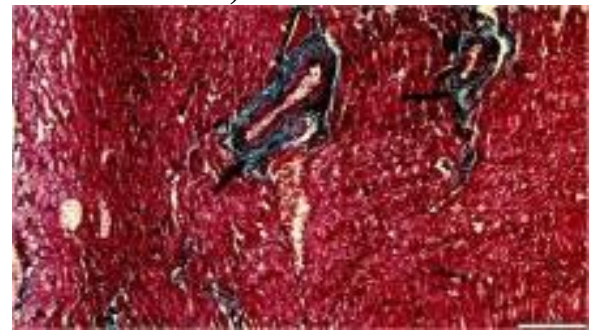

Fig.(9): A photomicrograph of a section of heart from group II (atherosclerotic group) showing dense collagen fibers (arrows) around the blood vessels.

(Masson's trichrome X400)

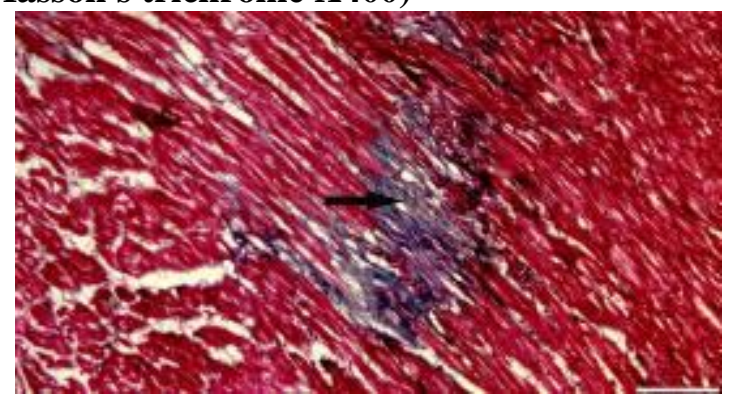

Fig.(10 ):A photomicrograph of a section of heart from group II showing dense collagen fibers(arrow) in between interrupted cardiac muscle fibers. (Masson's trichrome X400)

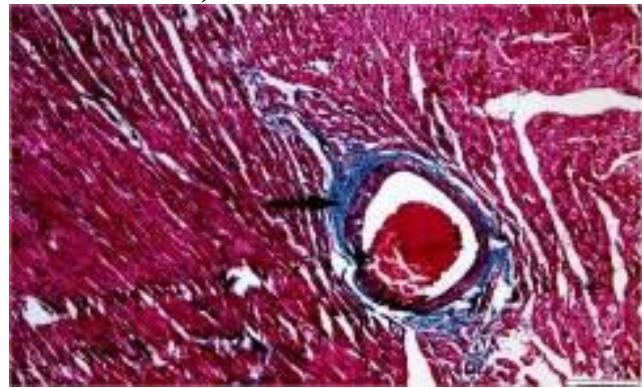

Fig.(34):A photomicrograph of a section of heart from group III (atorvastatin treated group) dense collagen fibers (arrow) around the blood vessels.

\section{(Masson's trichrome X400)}

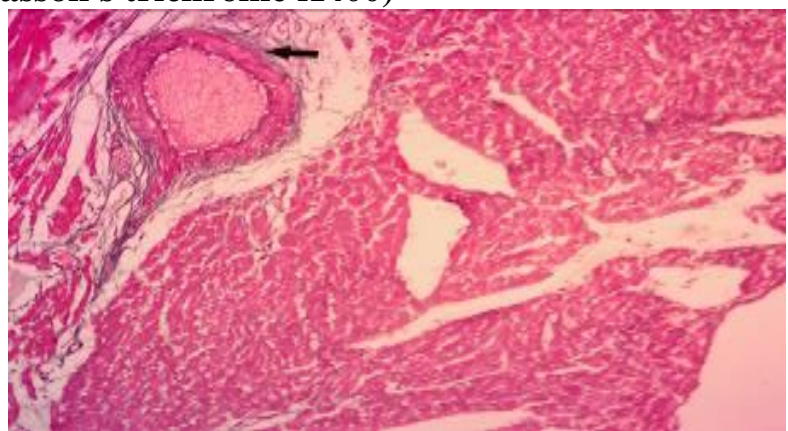

Fig.(35):A photomicrograph of a section of heart from group IV (curcumin treated group)showing minimal collagen fibers in between cardiac muscle fibers (arrow) (Masson's trichrome X400)

3- Troponin $\mathrm{T}$ immunostaining of cardiac muscle fibers:

\section{A. Control group}

Stained sections of cardiac muscle from group I (control group) showed cytoplasmic immunoexpersion of tropnin $\mathrm{T}$ in most cardiac muscle fibers (Fig.36).

\section{B. Atherosclerotic group}

The atherosclerotic group showed negative immuonexpression of troponin $\mathrm{T}$ in most of cardiac muscle fibers (Fig.37).

C. Atorvastatin treated group

Atorvastatin treated group showed mild immuonoexpression of troponin $\mathrm{T}$ in some cardiac muscle fibers (Fig.38). 


\section{Curcumin treated group}

Examination of a cardiac muscle sections from group IV (curcumin treated group)showed strong immunoexpersion of tropnin $\mathrm{T}$ in many cardiac muscle (Fig.39).

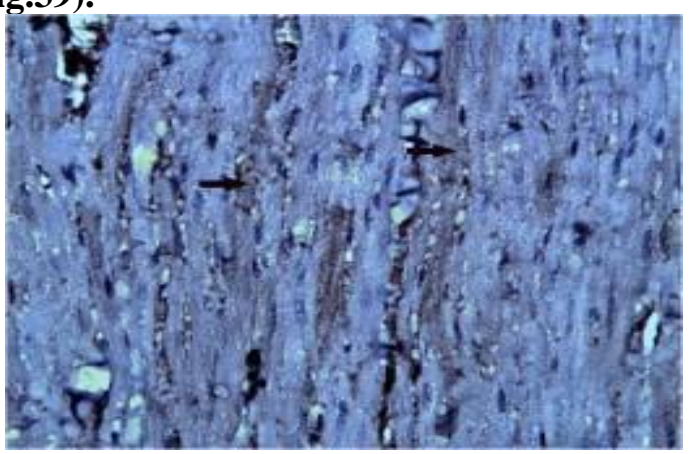

Fig.(36): A photomicrograph of a section of heart from group I (control group) showing brown immunoexpersion of tropnin $\mathrm{T}$ (arrows) in the cytoplasm of most cardiac muscle fibers .

\section{(Anti troponin $\mathrm{T} \times \mathbf{4 0 0}$ )}

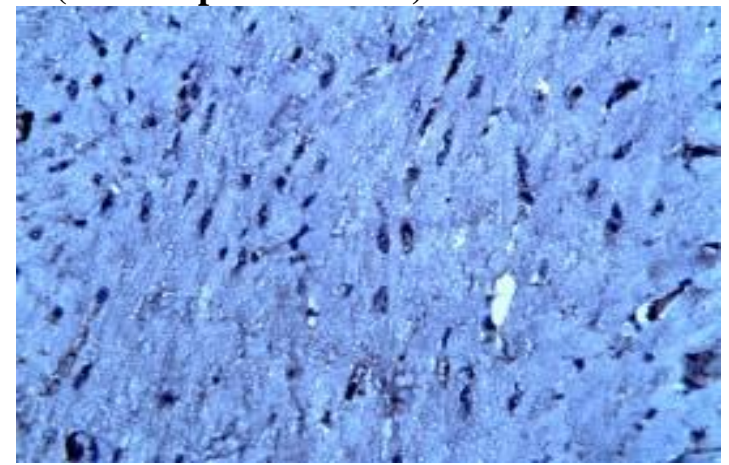

Fig.(37):A photomicrograph of a section of heart from group II (atherosclerotic group) showing negative immuonostaning of troponin $\mathrm{T}$ in most of cardiac muscle. (Antitroponin $\mathbf{T} \times \mathbf{4 0 0}$ )

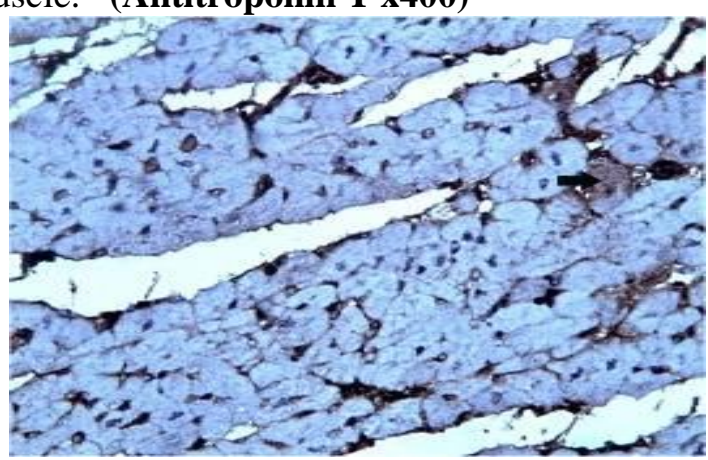

Fig.(38): A photomicrograph of a section of heart from group III (atorvastatin treated group) showing mild immuonoexpression of troponin $\mathrm{T}$ in some cardiac muscle (arrow).

(Anti troponin $\mathrm{T}$

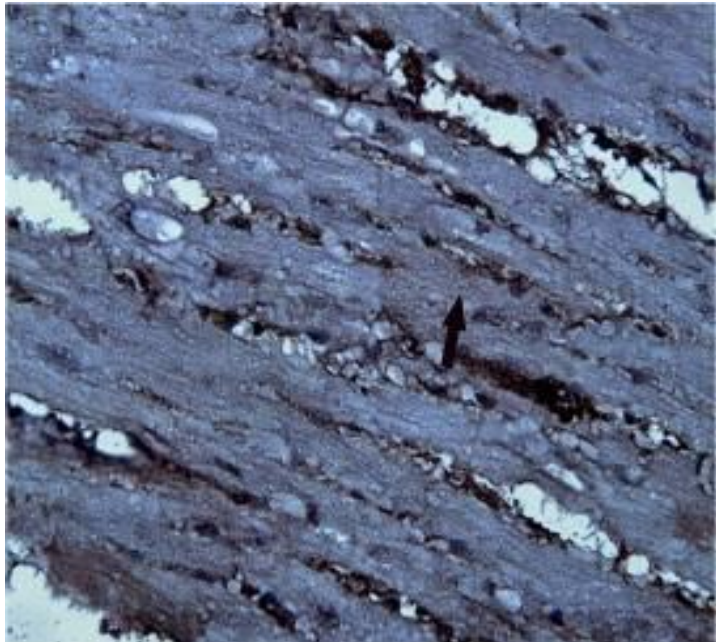

Fig. (11):A photomicrograph of a section of heart from group IV (curcumin treated group)showing positive immunoexpersion of tropnin $\mathrm{T}$ (arrow) in many cardiac muscle . (Anti troponin $\mathbf{T}$ $\mathrm{x400)}$

Morphometric results of cardiac muscle fibers

1- Mean area \%of collagen fibers in cardiac muscle fiber

The mean area \%of the collagen fibers deposition in cardiac muscle sections of group 1( control group ) was $2.922+1.41$.There was increase in the mean area $\%$ of collagen fibers atherosclerotic group , atorvastatin treated group and curcumin treated group $16.7+2.5,5.6+2.1$ and $3.4+0.8$ respectively (table $4)$.

There was significant increase in collagen fibers deposition in atherosclerotic and atorvastatin treated group in comparison to control group. There was non significant difference between curcumin treated group and control (Fig.40).

Table (4): The mean area $\%$ of collagen fibers in cardiac muscle in all studied groups

\begin{tabular}{|c|c|c|c|c|}
\hline & $\begin{array}{c}\text { Group } \\
\text { I }\end{array}$ & $\begin{array}{c}\text { Group } \\
\text { II }\end{array}$ & $\begin{array}{c}\text { Group } \\
\text { III }\end{array}$ & $\begin{array}{c}\text { Group } \\
\text { IV }\end{array}$ \\
\hline $\begin{array}{c}\text { Mean } \\
\text { area\% of } \\
\text { collagen } \\
\text { fibers in } \\
\text { cardiac } \\
\text { muscle }\end{array}$ & 2.922 & $16.7^{*}$ & $5.6^{*}$ & 3.4 \\
\hline
\end{tabular}
$\mathrm{x400)}$ 


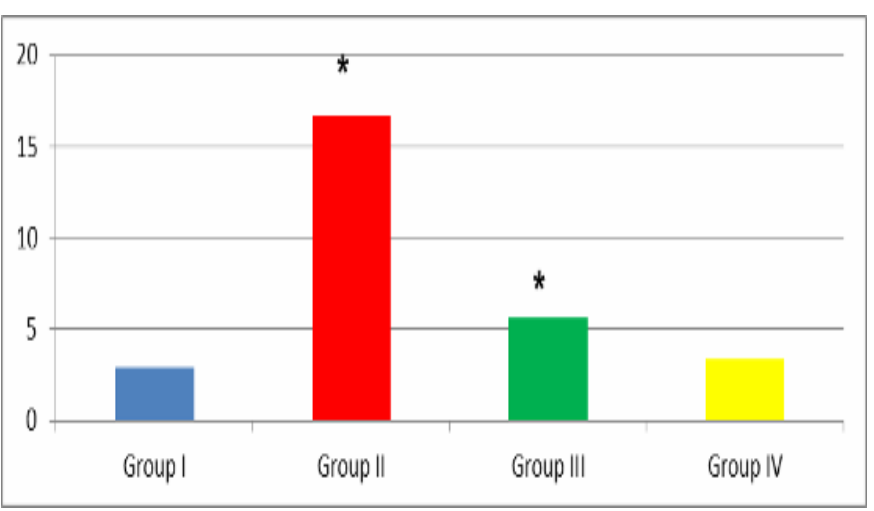

* Significant increase $(\mathrm{P}<0.05)$ compared to control

Fig. (12): Mean area \% of collagen fibers in cardiac muscle in all studied groups

\section{2- The mean area $\%$ of the immune reaction of troponin $\mathbf{T}$}

The mean area $\%$ of the immune positive cardiac muscle fibers stained by troponin $\mathrm{T}$ immuonohistochemical stain of group I (control group ) was $22.3+4$. There was decrease in the mean area $\%$ of troponin $\mathrm{T}$ immunopostive cardiac muscle fibers in atherosclerotic group, atorvastatin treated group and curcumin treated group $3.9+1,5.68+2.8$ and 10.3 +4.6 respectively (Table 5).

There was significant decrease in the mean area \% of the immune reaction of troponin $\mathrm{T}$ in group II and group III in comparison to control group. There are significant increase in the mean area $\%$ of the immune reaction of troponin $\mathrm{T}$ in group IV curcumin treated groups in comparison atorvastatin treated group (Fig. 41).

Table (5): The mean area \% immune reaction of troponin in all studied groups

\begin{tabular}{|c|c|c|c|c|}
\hline & $\begin{array}{c}\text { Group } \\
\text { I }\end{array}$ & $\begin{array}{c}\text { Group } \\
\text { II }\end{array}$ & $\begin{array}{c}\text { Group } \\
\text { III }\end{array}$ & $\begin{array}{c}\text { Group } \\
\text { IV }\end{array}$ \\
\hline $\begin{array}{c}\text { Mean } \\
\text { area\% } \\
\text { immune } \\
\text { reaction } \\
\text { of } \\
\text { troponin }\end{array}$ & 22.3 & $3.9^{*}$ & $5.68^{*}$ & $10.3^{*}$ \\
\hline
\end{tabular}

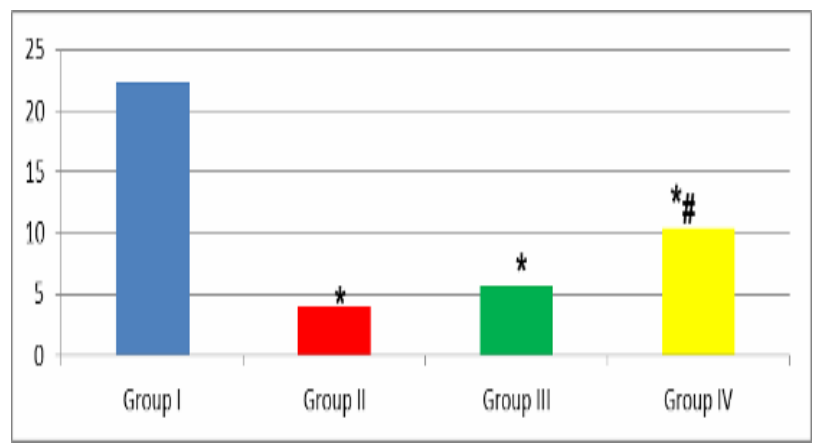

* Significant decrease $(\mathrm{P}<0.05)$ compared to control

*\#Significant increase $(\mathrm{P}<0.05)$ compared to group III
Fig. (13):Mean area\% immune reaction of troponin in the cardiac muscle in all studied groups

\section{DISCUSSION}

The objective of this study was to establish animal models of experimentally induced atherosclerosis to evaluate and to compare the possible therapeutic effect of both atorvastatin and curcumin. The current study recorded that statin and curcumin play a crucial role in management of atherosclerosis. In view of their anti inflammatory properties, immunomodulatory effect and anti oxidant effect, either atorvastatin or curcumin potentially represents novel treatment modality for atherosclerosis.

The model of induced of atherosclerosis through Cholesterol powder $1 \%$ mixed with ordinary diet for a period of 8 weeks ${ }^{(14)}$.

In the present study, the related changes in the aorta and cardiac muscle fibers were investigated in four groups of rabbits, these were group I(control), group II(atherosclerotic ), group III (atorvastatin treated group ) and group IV(curcumin treated group).

\section{In aortic sections}

In Hx. and $\mathbf{E}$ stained sections, the current study showed control group with intact tunica intima, thin wavy corrugated endothelium, thin sub endothelial layer and apparent wavy corrugated internal elastic fibers. While, atherosclerotic group revealed tunica intima with lost corrugation and apparent bulge formation. There were adhesions of RBCs on the surface of the intima. The intima showed foam cell in the sub endothelial layer occupied by fatty material. There was a significant increase in the mean thickness of both tunica intima and media of thoracic aorta in atherosclerotic group when compared to others groups.

These findings could be attributed to the effect of atherosclerotic agent (cholesterol powder) resulting in large areas of endothelial cell injury. This was in concomitant with Naito et al. (14) who reported the same histopathological features of rabbits received Cholesterol powder $1 \%$ mixed with ordinary diet for a period of 8 weeks. This was due to inability of vascular endothelium to repair large areas of endothelial cell injury in atherosclerosis. Smooth muscle cells of the underlying medial layer are capable of forming a thinner pseudoendothelium lining the luminal surface. Pseudoendothelial smooth muscle cell has an inhibitory influence on vascular endothelium regrowth.

In $\mathbf{H} \mathbf{x}$. and $\mathbf{E}$ stained aortic sections of atorvastatin treated group (group III) the tunica intima was intact with thin wavy corrugated endothelium while some areas possessed absence of such corrugation. Thin sub endothelial layer and apparent wavy corrugated internal elastic lamina. Although there was a significant increase of the mean thickness of both tunicae intima \& media of atorvastatin treated group 
versus the control yet it showed a significant decrease compare with the atherosclerotic group. This could be explained by Nalini et al. ${ }^{(10)}$ who proved that the atorvastatin is anti-inflammatory, incite reduction of the necrotic plaque core, and improve endothelial function, leading to plaque stabilization and, sometimes, plaque regression.

In $\mathbf{H} \mathbf{x}$. and $\mathbf{E}$ stained aortic sections of curcumin treated group (group IV), the tunica intima was intact with thin wavy corrugated endothelium, thin sub endothelial layer and apparent wavy corrugated internal elastic fibers. There were significant decrease in the thickness both tunica intima and media in curcumin treated groups in comparison to atherosclerotic group.

This was in agreement with Shoba et al. ${ }^{(17)}$ who reported that intake of antioxidants, like curcumin had a beneficial effect on atherosclerosis, contributing to reduce the degree of evolution of the atherosclerotic lesion, reduction of serum and liver cholesterol levels, increase of cholesterol and triacylglycerol fecal excretion, resulting in a greater beneficial effect on atherosclerosis than statin. On the other hand Bustos et al. (18) found that the effects of statins involve improving endothelial function, enhancing the stability of atherosclerotic plaques, decreasing oxidative stress and inflammation and inhibiting the thrombogenic response ${ }^{(\mathbf{1 9})}$.

In orcein stained aortic sections of the control group showed prominent dark brown wavy internal elastic lamina. Tunica media appeared rich in elastic fibers. These fibers were parallel in distribution while atherosclerotic group there was disruption of the internal elastic lamina. Tunica media possessed areas with complete loss of elastic fibers. Other areas showed irregularly condensed elastic fibers towards the tunica adventitia. There was a significant decrease in the mean area\% of elastic fibers versus the control.

While in Atorvastatin and curcumin treated groups there were prominent internal and external elastic laminae. Tunica media appeared rich in parallel elastic fibers. Small areas showed lost elastic fibers. There was a significant decrease in the area $\%$ of elastic fibers of atrovastain treated group versus the control, meanwhile this was a non-significant difference in curcumin treated group compared to the control group.

This was in agreement with Ahmed et al. ${ }^{(20)}$, who explained that decrease of elastin synthesis by smooth muscle cells was a result of oxidative damage of medial smooth muscle cells in atherosclerosis. In the current study, the relative increase in elastic fibers in curcumin treated group might be explained by Yang et al. (21), who proved that the significant increase of intimal and medial elastic fiber content in treated group could be due to the antioxidant effect of which protects smooth muscle cells in the media against the oxidative damage in atherosclerosis leading to increase of elastin synthesis.

\section{Masson's trichrome stained aortic sections:}

In Masson's trichrome stained sections of a thoracic aorta of Atherosclerotic group showed smooth muscle fibers present in the tunica intima, most probably migrated from the tunica media. There was decrease in amount of smooth muscles in the tunica media and disorganized elastic fibers. This was in agreement with Dick et al. (22) who found that the increased accumulation of lipids in the sub intima as well as increased contraction, migration and proliferation of vascular smooth muscle cell fibers and damage of smooth muscle cells by free radical in the media in atherosclerosis lead to migration of vascular smooth muscle from the media to the intima. Although in the Atorvastatin treated group some smooth muscle fibers were present in the tunica intima. The tunica media possessed wavy elastic fibers and smooth muscle fibers distributed in between. This could be due to the curative effect of atorvastatin and might be explained Krempf et al. ${ }^{(23)}$, who stated that atorvastatin has antioxidant and anti-inflammatory actions.

While in curcumin treated group the tunica media showed wavy elastic fibers and smooth muscle cells distributed in between. This was in agreement with Jawien et al. (24) who showed that the antioxidant effect of curcumin protects the smooth muscle cells against damage by free radicals inhibits lipid peroxidation of cell membrane (membrane stabilizer). Such inhibition may be through its action inside the cell by neutralizing and savenging free radicals or it might prevent the oxidative damage to the cell membrane through preventing the oxidation of polyunsaturated fatty acids.

Endothelial cells (EC) have many functions and play a central role in control of coagulation, thrombolysis, vascular tone, permeability, inflammation, tissue repair and angiogenesis. They constitute a heterogeneous cell population in the human body. The molecular characteristics of EC vary along the vascular tree and in the same organ between different vessels. The immunohistochemical expression pattern of CD34 is commonly used as EC markers on normal endothelium ${ }^{(25)}$.

In our study CD34 immunohistocemical stained aortic sections in the control group recorded few positive brownish cytoplasmic reaction confined to endothelial cells while in Atherosclerotic group there was significant increase in the mean area $\%$ of the immune reaction of CD34 in comparison to control group. This can be explained by LDL oxidation in atherosclerosis which enhances the expression of CD34 that influences the adhesion and endothelial transmigration of monocytes. 
Furness and McNagny (26) explained that angiogenesis is the capillary growth from existing vessel. The term also involves division of existing vessel by transendothelial cell bridges or pillars of peri endothelial cells. Angiogenesis is intiated by hypoxia -inducible factor (HIF).This leads to a local up regulation in expresion of vascular endothelial growth factor (VEGF) an a number of other angiogenic factors.

In contrary with Krempf $\boldsymbol{e t}$ al. ${ }^{(23)}$, who found that atorvastatin has high peroxidase and acid phosphatase activity, and an antioxidant and anti-inflammatory actions, which protects the endothelial cells against injury.

While in curcumin treated group, few CD34 immunoreactive was detected in cytoplasm of endothelial cells and there was a significant decrease in the mean area \% of the immune reaction of CD34 versus atherosclerotic and atorvastatin treated groups. This was in agreement with Motterlini et al. ${ }^{(25)}$ who showed that curcumin seemed to inhibit monocyte adhesion by increasing the resistance of LDL to oxidation.

\section{In cardiac muscle sections}

Hx. and E stained sections

The control group showed branching cardiac cell with centrally located nuclei. Each muscle fiber is surrounded with endomysium containing normal blood vessels, which possessed patent lumen, without any narrowing by atheromatous plaque. While atherosclerotic group showed many muscle fibers with pale cytoplasm and darkly stained peripheral nuclei. Many cardiac muscle fibers appeared darkly acidophilic with darkly stained nucleus, while others posses apoptotic change. There was mononuclear infiltration, thickened media of a blood vessel with formation of bulge to words the lumen to which RBCs were stuck.

This was in concomitant with Takashi et al. (38) who found numerous hyaline microthrombi were observed in the intramural arterioles and capillaries, consisting of enlarged endothelial cells located adjacent to or within central hemorrhagic and fibrotic foci and negative for phosphotungstic acidhematoxylin stain.

Curcumin treated group showed many healthy branching cardiac muscle fibers with pale central nucleus. Many cardiac muscle fibers possessed pale cytoplasm with absent nuclei, while others showed vacuolated cytoplasm with small dark. RBCS were stuck on the lumen of blood vessel. This in agreement with Olszaneckir $\boldsymbol{e t} \boldsymbol{a l}^{\left({ }^{(28)}\right.}$ who showed that Ingredients like Curcuma longa L. (Zingiberaceae) exhibits anti-inflammatory and antithrombogenic activities.

\section{Masson's trichrome stained cardiac muscle section}

In Control group revealed minimal collagen fibers deposition around the blood vessels. While in Atherosclerotic group there was dense collagen fibers deposition around blood vessels and in between interrupted cardiac muscle fibers. There was a significant increase in collagen content in atherosclerotic group compared to control group which can be explained by migration and proliferation of smooth muscle cells to the intima from the media of cardiac blood vessel leading to an increase in collagen synthesis around blood vessel and in between cardiac muscle. Similar findings were observed by Takashi $\boldsymbol{e t}$ al. ${ }^{(27)}$ who found numerous areas of hemorrhage and fibrosis were observed throughout the left and right ventricular and atrial myocardia, without any evidence of regional predominance. Several areas of spotty fibrotic foci were visualized using Masson's trichrome stain. In addition, fibrotic foci with fibroblastic proliferation, interstitial edema, and lymphoplasmacytic infiltration were observed. In Atorvastatin treated group there was dense collagen fibers deposition around blood vessels. This was in concomitant with Results from Olszaneckir $\boldsymbol{e t}$ al. ${ }^{(28)}$ who showed that hypoxia and stretched myocardial wall leads to production of transforming growth factor (TGF) which plays an important role in collagen production. Over expression of TGF in heart leads to cardiac hypertrophy and interstitial fibrosis. On the other hand, Dongdan et al. ${ }^{(29)}$ reported that Statins, a potent lipid-modified agent, has potent effects on protecting endothelium through multiple mechanisms in the early stage of dyslipidemia, initiating high-dose of statins therapy might promptly prevent endothelial deterioration.

But in curcumin treated group there was minimal collagen fibers deposition in between cardiac muscle fibers. Non significant difference between curcumin treated group and control group. There was significant decrease in collagen fibers deposition in curcumin treated group in comparison to atorvastatin. That could be explained by antioxidant effect of curcumin which inhibits the fibrotic activity through significant reduction of the elevated serum level of P-III-P (Procollagen III - peptide), marker of fibro genesis ${ }^{(29)}$.

This is in agreement with Jawien et al. ${ }^{(27)}$ who showed that curcumin extract could reduce the elevated expression of NFKB and TNF- $\alpha$ in rabbit. The activation of NF- $\mathrm{KB}$ is linked to a variety of inflammatory diseases, including atherosclerosis, myocardial infarction, cancer, diabetes, allergy, asthma, arthritis, Crohn's disease, multiple sclerosis, Alzheimer's disease, osteoporosis, psoriasis, septic shock, and AIDS.

Troponin $\mathbf{T}$ immunostaining of cardiac muscle:

Troponin $T$ proved to be a very useful marker in the diagnosis of acute myocardial damage which had nearly absolute myocardial tissue specificity as well as 
high sensitivity. it is currently the gold standard in the diagnosis of myocardial infarction ${ }^{(30)}$.

Stained sections of cardiac muscle from group I (control group) showed immunoexpersion of tropnin $\mathrm{T}$ in most cardiac muscle fibers while in atherosclerotic group, there was negative immuonexpression of troponin $\mathrm{T}$ in most of cardiac muscle fibers. This was in agreement with Cina et al. (31) who confirmed the diagnostic efficacy of this marker as a valid, highly sensitive indicator of infarct damage.

In Atorvastatin treated group; there was mild immuonoexpression of troponin $\mathrm{T}$ in some cardiac muscle fibers. This was in concomitant with Järvenpää et al. ${ }^{(32)}$ who showed that Troponin $\mathrm{T}$ loss of staining in preinflammatory lesions however, nonspecific patchy loss of staining was present in negative controls and in viable myocardium and observed that in cases with coronary atherosclerosis but no clear signs of acute ischemic damage, the comparative analysis of histologic and immunohistochemical methods can detect ischemic areas and/or latent necrosis of the myocardium.

While in curcumin treated group there was strong immunoexpersion of tropnin $\mathrm{T}$ in many cardiac muscles. This was in concomitant with Carluccio et al. (33) who showed that the antioxidant effect of curcumin enhance cardiac fibroblast which regulate extracellular matrix (ECM) by synthesis of matrix molecules and secretion of the ECM -degrading enzyme metalloporteinases (MMPs).

\section{CONCLUSION}

Curcumin has potential role in treatment of atherosclerosis and myocardial infarction.

\section{REFERENCES}

1.Herrington W, Lacey B, Sherliker P, Armitage J, Lewington S (2016): Epidemiology of atherosclerosis and the potential to reduce the global burden of atherothrombotic disease. Circ. Res., (118): 535-546.

2. Caleb EF (2011): Atherosclerosis is an old disease: Summary of the Ruffer Centenary Symposium, The Paleocardiology of Ancient Egypt, a meeting report of the Horus Study team. Experimental Gerontology, 46(11): 843-846.

3. Bural GG, Torigian DA, Chamroonrat W, Houseni M, Chen W, Basu S, Kumar R and Alavi A (2008): FDGPET is an effective imaging modality to detect and quantify age-related atherosclerosis in large arteries. European Journal of Nuclear Medicine and Molecular Imaging, 35(3): 562-569.

4. Fumiyuki I, Kusaiaziz GS , Sergo W (2006): Update on Coronary Angioscopy: Review of a 20-Year Experience and Potential Application for Detection of Vulnerable Plaque. Journal of Interventional Cardiology, 19(1): 1725 .
5. Matthew J and Sorrentino A (2011): Drug Therapy for Dyslipidemia. Hyperlipidemia in Primary Care. Current Clinical Practice, 121-13.

6. Teiten MH, Dicato $M$ and Diederich $M$ (2014): Hybrid Curcumin Compounds: A New Strategy for Cancer Treatment. Molecules, 19: 20839-63.

7. Kapakos G, Youreva V and Srivastava AK (2012): Cardiovascular protection by curcumin: molecular aspects. Indian J Biochem Biophys., 49(5):306-15.

8. Nasiripour S, Gholami Kh, Mousavi S, Mohagheghi A, Radfar M, Abdollahi M, Khazaeipour $\mathbf{Z}$, Mojtahedzadeh M (2014): Comparison of the effects of enoxaparin and heparin on inflammatory biomarkers in patients with st-segment elevated myocardial infarction: a prospective open label pilot clinical trial. Iran. J. Pharm. Res., 13: 583-590.

9. Yuan HY, Kuang SY, Zheng X, Ling HY, Yang Y, Yan P, Kai L, Duan-Fang L (2008): Curcumin inhibits cellular cholesterol accumulation by regulating SREBP-1/caveolin-1 signaling pathway in vascular smooth muscle cells. www.chinaphar.com/article/view/4872/10431

10. Nalini MR, Malayannan S, Margaret S, Thomas CS, Marek N, Joseph PM, Ravinder JS, Neil JS, Robert OB, Thomas CS (2010): Atorvastatin Inhibits Hypercholesterolemia-Induced Cellular Proliferation and Bone Matrix Production in the Rabbit Aortic Valve. Basic Science Reports. Circulation, 105: 26602665.

11. Joanna L, Izabela K, Jan B (2008): The use of botanical microtechnique paraffin in anatomical studies of lichens. Annales UMCS, Biologia, 63 (1): 36-42.

12. Godwin A (2011): Histochemical Uses of Haematoxylin - A Review. JPCS., 1: 24-34.

13. Beecken H, Gottschalk E, Gizycki U et al. (2003): "Orcein and litmus". Biotechnic \& Histochemistry, 78 (6): 289-302.

14. Lamar M and Jones BS (2010): ASCP Mastering the Trichrome Stain Connection. J Clin Pathol .53(10):756-759.

15. Nielsen JS, McNagny KM (2008): Novel functions of the CD34 family. J of Cell Science., 121 (Pt 22): 36823692.

16. Naito $M$, Nomura $H$, Esaki $T$ et al. (2008): Characteristics of macrophage-derived foam cells isolated from atherosclerotic lesions of rabbits.. Histol Histopathol., 13: 797-817.

17. Shoba G, Joy D, Joseph T, Majeed M, Rajendran R, Srinivas PS (1998): Influence of piperine on the pharmacokinetics of curcumin in animals and human volunteers. Planta Med., 64:353-356.

18. Bustos C, Hernandex-Presa MA, Ortego $M$ et al. (2008): HMG-CoA reductase inhibition by atorvastatin reduces neointimal inflammation in a rabbit model of atherosclerosis. J Am Coll Cardiol., 32: 2057-2064.

19. Dugasani S, Pichika MR, Nadarajah VD, Balijepalli MK, Tandra S, Korlakunta JN(2010): Comparative antioxidant and anti-inflammatory effects of [6]gingerol, [8]-gingerol, [10]-gingerol and [6]-shogaol and Statin., 127: 515-20

20. Ahmed RS, Seth V, Banerjee BD (2000): Influence of dietary ginger (Zingiber officinales Rosc) on 
antioxidant defense system in rabbit: Comparison with statin. Indian J Exp Biol., 38: 604-6.

21. Yang CH, Yue J, Sims M, Pfeffer LM (2013): The curcumin analog EF24 targets NF- $\mathrm{BB}$ and miRNA-21, and has potent anticancer activity in vitro and in vivo. PloS one, 8(8):e71130.

22. Dick W, Valentina $P$, Roeland $H$, Kjell $H$, Ruud AB, Anders F, Jan HN(2013): Impaired Collagen Biosynthesis and Cross- linking in Aorta. Vascular Heart Disease. J Am Heart Assoc., 2: 57-61.

23.Krempf M, Rohmer V, Springer TA (2000): Efficacy and safety of micronized fenofibrate in a randomized double-blind study comparing four doses from $200 \mathrm{mg}$ to $400 \mathrm{mg}$ daily with placebo in patients with hyper cholesterolemia. Diabetes Metabolism, 26(3): 184-191.

24.Jawien J, Gajda M, Mateuszuk L, Olszanecki R, Jakubowski A, Szlachcic A et al. (2005): Inhibition of nuclear factor-kappaB attenuates artherosclerosis in apoE/LDLR - double knockout mice. JPhysiol Pharmacol., 56:483-489.

25. Motterlini R, Foresti R, Bassi R, Green CJ (2000): Curcumin, an antioxidant and anti-inflammatory agent, induces heme oxygenase- 1 and protects endothelial cells against oxidative stress. Free Radic Biol Med., 28:13031312.

26. Furness SG, McNagny K (2006): Beyond mere markers: functions for CD34 family of sialomucins in hematopoiesis. Immunol. Res., 34 (1): 13-32.

27. Takashi T, Sohsuke Y, Atsunori N, Hirotsugu N, Aya N, Masanori H, Yasuyuki S and Toshiyuki N (2015): An autopsy case of myocardial infarction due to idiopathic thrombotic thrombocytopenic purpura. Diagnostic Pathology, 26, 10: 52.

28. Olszaneckir R, Jawien J, Gajda M, Matuszuk E, Gepska A, Korapiowska M, Korbut R (2005): Effect of curcumin on atherosclerosis in apoE/LDLR - Double Knockout Mice. Journal of Physiology and Pharmacology, 56(4): 627-635.

29. Dongdan Z, Qing L, FanFang Z, Zhuocheng M, Anping C, Ruofeng Q, Rulin X, Dongjuan L, Weiyi $M$ (2015): Atorvastatin protects endothelium by decreasing asymmetric dimethylarginine in dyslipidemia rats. Lipids in Health and Disease, 14: 41.

30. Monaco C, Paleolog E (2004): Nuclear factor kappaB: a potential therapeutic target in atherosclerosis and thrombosis. Cardiovasc Res., 61:671-682.

31. Cina SJ, Brown DK, Smialek JE, Collins KA (2001): A rapid postmortem cardiac troponin $T$ assay: laboratory evidence of sudden cardiac death. Am J Forensic Med Pathol., 22(2):173-6.

32. Järvenpää ML, Kuningas $K$, Niemi I, Hedberg P, Ristiniemi N, Pettersson K, Lövgren T (2012): Rapid and sensitive cardiac troponin I immunoassay based on fluorescent europium (III)-chelate-dyed nanoparticles. Clin Chim Acta., 414:70-5.

33. Carluccio MA, Siculella L, Ancora MA, Massaro M, Scoditti E, Storelli C et al. (2003): Olive oil andred wine antioxidant polyphenols inhibit endothelial activation: antiatherogenic properties of Mediterranean diet phytochemicals. Arterioscler Thromb Vasc Biol., 23:622-629. 